\title{
Enhancement of ovalbumin-specific IgA responses via oral boosting with antigen co- administered with an aqueous Solanum torvum extract
}

\begin{abstract}
An experiment was conducted with the objective to enhance mucosal immunity against ovalbumin (OVA) by co-administration of OVA with an aqueous extract from the fruit of Solanum torvum (STE). Five groups of female ICR mice aged approximately 8 weeks at the commencement of the experiment were caged in groups of eight and received various treatments. The treatments included OVA alone, OVA with cholera toxin (CT), and OVA with various doses of STE. Mice were primed intraperitoneally with $500 \mathrm{\varepsilon g}$ of OVA alone or co-administered with $0.1 \varepsilon \mathrm{g} \mathrm{CT}$, or with 1 हg STE. All mice were boosted orally via gastric intubation 14 days after priming with $10 \mathrm{mg}$ OVA alone, or co-administered with $10 \mathrm{\varepsilon g}$ CT or with $10 \mathrm{mg}, 1 \mathrm{mg}$ or $0.1 \mathrm{mg}$ STE. One week later all mice were killed and organs obtained for analysis of the immune response. Intestinal, faecal and pulmonary OVA-specific sIgA concentration was significantly increased $(\mathrm{p}<0.05)$ in mice that received booster combinations of OVA/CT and OVA with all extract doses $(\mathrm{p}<0.05)$. Specific serum IgG titres did not differ significantly between groups. It is concluded that STE can significantly enhance secretory immunity in the intestine to OVA with mucosal homing to the lungs. The adjuvant effect of STE is comparable to that of CT.
\end{abstract}

Keyword: Solanum torvum; IgA; Oral adjuvant; Ovalbumin 